\title{
What is the role of bronchoalveolar lavage in the diagnosis of COVID-19?
}

\author{
Bernadette Corica $^{1}$ - Giovanni Talerico ${ }^{2}$ - Giulio Francesco Romiti ${ }^{1}$ (i)
}

Received: 30 April 2021 / Accepted: 4 May 2021 / Published online: 17 May 2021

(c) Società Italiana di Medicina Interna (SIMI) 2021

Since the beginning of the COVID-19 pandemic, the diagnosis of SARS-CoV-2 infection has represented one of the most significant areas of uncertainty. The need for prompt isolation and tracking of suspected cases led to extensive use of the real-time polymerase chain reaction (RT-PCR) performed on nasopharyngeal swabs (NPS), which was proposed and adopted immediately as the cornerstone in the diagnosis of COVID-19 [1]. However, poor knowledge of the chances of false-negative and false-positive results [2], and misleading evaluation of post-test probability [3], often resulted in doubts and uncertainties in the diagnostic workup of suspected COVID-19 infection-especially in those patients who had very low or very high pre-test probability of the disease.

After the initial phase of this pandemic, in fact, some author argued for low sensitivity of RT-PCR performed on NPS [2], suggesting that repeating the test, or using other samples, may reduce the number of false negatives [4], which another study estimated to be as high as 9.3\% [5]; noteworthy, other reports found even lower sensitivity for NPS [6, 7]. Beyond these data, the overarching question still remains unsolved: how much can we rely on a single negative NPS to rule out COVID-19, if clinical suspicion remains high?

Clinical test performance of RT-PCR in different biological specimens is variable, and depends on both the analytical sensitivity and the patient-specific pre-test probability, which is in turn influenced by several factors, including exposure history, clinical presentation, and disease prevalence [8]. Suboptimal sample collection [9], the timing of

Giulio Francesco Romiti

giuliofrancesco.romiti@uniroma1.it

1 Department of Translational and Precision Medicine, Sapienza-University of Rome, Viale del Policlinico 155, 00161 Rome, Italy

2 Internal Medicine Unit, Policlinico Casilino, Rome, Italy the diagnostic test [10], and viral load [11] also affect test performance.

To overcome the limitations of the NPS test, performing RT-PCR on other biological samples has been repeatedly postulated and proposed to improve our diagnostic ability [6]. Bronchoalveolar lavage (BAL) has been identified as an interesting candidate to increase diagnostic yield, especially in those patients with highly suspected clinical presentation and negative NPS test [6]. However, conflicting evidence has emerged on the role of BAL $[12,13]$, and although the Infectious Disease Society of America (IDSA) currently recommend collecting lower respiratory tracts specimen (including BAL) in patients with a first negative test and a high suspicion of disease [14], further evidence is highly required and needed on the topic.

The study of Barberi and colleagues [15] fits perfectly into this context and may provide further insights on the issue. In this retrospective analysis, 198 subjects with at least one negative NPS underwent RT-PCR on BAL to detect SARS-CoV-2. The authors found $16 \%$ of patients with positive RT-PCR on BAL, with isolation of other pathogens (mostly Fungi) in 33\% of the subjects; moreover, they recorded a low rate of procedure-related complications.

Beyond the obvious limitations (mainly represented by the retrospective design, the low number of patients who underwent repeated NPS before the inclusion, and the physician-based indication for performing BAL), the study allows some considerations.

First, although speculative, the study demonstrates how performing BAL can be helpful to correctly diagnose COVID-19 in a significant proportion of patients, in whom the diagnosis would have been missed otherwise. Though it is unclear how many subjects would have been diagnosed by simply repeating NPS, the added value of BAL may be particularly relevant, since it may directs physicians towards correct treatment and isolation of patients, and may contributes to the prevention of disease spreading, especially in hospital settings. On the other side, 
performing BAL may be challenging in some scenarios, including centres with limited facilities and expertise, and patients with severe respiratory failures. In these cases, the potential added value of BAL should be carefully weighted against the logistic constraints, and a multidisciplinary approach may be recommended to decide whether or not referring the patient for the procedure.

The study also showed that roughly one-third of BAL resulted positive for other microorganisms, with unclear clinical significance: for example, isolation of low fungal load in BAL, especially in immunocompetent patients, should be evaluated carefully according to the pre-test probability for fungal infections, to avoid the risk of overdiagnosis and overtreatment $[16,17]$. This also applies to other clinical scenarios, and reinforces the importance of critical reasoning on test results, accounting for the posttest probabilities rather than simply relying on the "positive" or "negative" result. Although BAL may provide valuable diagnostic informations, caution should be used, and results should be discussed with an infectious disease specialist, especially when doubts arise and the interpretation is not straightforward.

Taken these findings together, some questions remain open. What is the role of BAL in this pandemic? When should we perform a BAL?

While the study of Barberi may not provide definitive answers to these doubts, it seems to support the current IDSA recommendation on testing those patients with high clinical suspicion and negative NPS. Identifying those patients who may benefit most from BAL testing may also be helpful to reduce unnecessary costs and ensure effective resource allocation, although limited evidence currently exists. In their analysis, the authors found some variables associated with an increased likelihood of BAL positivity, including CT characteristics [15], but these data are preliminary, and need confirmation on larger prospective cohorts. Further studies are needed to define the role of BAL, and which subgroup of suspected COVID-19 patients represents the optimal population to test with BAL.

From a broader perspective, these data remind us of the uncertainty that still surrounds this pandemic, and emphasize the importance of the correct interpretation of diagnostic tests, particularly in the view of pre-test and post-test probabilities.

Diagnosis, a central moment in medical practice, is a complex and integrated process, that cannot (and must not) be reduced to a single test's result. Accounting for the diagnostic performance, as well as for the overall probability of the disease, is the key to a balanced interpretation of new data acquired during the diagnostic pathways. While these basic concepts may sound obvious, they were often overlooked and forgotten during this pandemic [3].
While the role of BAL in the approach to COVID-19 is still not written in the stone, we should take this opportunity to learn from the conundrums that this pandemic keeps throwing at us. Looking at the patients before the tests will help us go further when the "diagnostic road" will become bumpy, but will also remind us to avoid unnecessary tests (or interventions) when there is no need: see the forest for the trees must remain our goal, during and after this pandemic.

\section{Declarations}

Conflict of interest The authors have nothing to disclose.

Statement of human and animal rights This article does not contain any studies with human participants or animals performed by any of the authors.

Informed consent Not applicable.

\section{References}

1. Cheng MP, Papenburg J, Desjardins M et al (2020) Diagnostic testing for severe acute respiratory syndrome-related Coronavirus 2: a narrative review. Ann Intern Med 172(11):726-734. https:// doi.org/10.7326/M20-1301

2. Mina MJ, Parker R, Larremore DB (2020) Rethinking COVID19 test sensitivity - a strategy for containment. N Engl J Med 383(22):e120. https://doi.org/10.1056/nejmp2025631

3. Romiti GF, Talerico G (2021) Embracing the uncertainty: an important lesson from COVID-19. J Gen Intern Med. https://doi. org/10.1007/s11606-021-06809-2 (Published online April 26, 2021)

4. Dramé M, TabueTeguo M, Proye E et al (2020) Should RT-PCR be considered a gold standard in the diagnosis of COVID-19? J Med Virol 92(11):2312-2313. https://doi.org/10.1002/jmv.25996

5. Kanji JN, Zelyas N, MacDonald C et al (2021) False negative rate of COVID-19 PCR testing: a discordant testing analysis. Virol J 18(1):13. https://doi.org/10.1186/s12985-021-01489-0

6. Wang W, Xu Y, Gao R et al (2020) Detection of SARS-CoV-2 in different types of clinical specimens. JAMA. https://doi.org/10. 1001/jama.2020.3786 (Published online March 11, 2020)

7. Clerici B, Muscatello A, Bai F et al (2021) Sensitivity of SARS$\mathrm{CoV}-2$ detection with nasopharyngeal swabs. Front Public Heal 8(January):1-5. https://doi.org/10.3389/fpubh.2020.593491

8. Sethuraman N, Jeremiah SS, Ryo A (2020) Interpreting diagnostic tests for SARS-CoV-2. JAMA J Am Med Assoc 323(22):22492251. https://doi.org/10.1001/jama.2020.8259

9. Kinloch NN, Ritchie G, Brumme CJ et al (2020) Suboptimal biological sampling as a probable cause of false-negative COVID-19 diagnostic test results. J Infect Dis 222(6):899-902. https://doi. org/10.1093/infdis/jiaa370

10. Kucirka LM, Lauer SA, Laeyendecker O, Boon D, Lessler J (2020) Variation in false-negative rate of reverse transcriptase polymerase chain reaction-based SARS-CoV-2 tests by time since exposure. Ann Intern Med 173(4):262-267. https://doi.org/ 10.7326/M20-1495 
11. He X, Lau EHY, Wu P et al (2020) Temporal dynamics in viral shedding and transmissibility of COVID-19. Nat Med 26(5):672675. https://doi.org/10.1038/s41591-020-0869-5

12. Salton F, Geri P, Confalonieri M (2020) Factors limiting the utility of bronchoalveolar lavage in the diagnosis of COVID-19. Eur Respir J. https://doi.org/10.1183/13993003.03383-2020

13. Geri P, Salton F, Zuccatosta L et al (2020) Limited role for bronchoalveolar lavage to exclude COVID-19 after negative upper respiratory tract swabs: a multicentre study. Eur Respir J. https:// doi.org/10.1183/13993003.01733-2020

14. Hanson KE, Caliendo AM, Arias CA et al (2021) The infectious diseases Society of America Guidelines on the diagnosis of COVID-19: molecular diagnostic testing. Clin Infect Dis. https:// doi.org/10.1093/cid/ciab048 (Published online January 22, 2021)

15. Barberi C, Castelnuovo E, Dipasquale A et al (2021) Bronchoalveolar lavage in suspected COVID-19 cases with a negative nasopharyngeal swab: a retrospective cross-sectional study in a high-impact Northern Italy area. Intern Emerg Med. https://doi. org/10.1007/s11739-021-02714-y (Published online March 26, 2021)

16. Shah AS, O'Horo JC, Tang S, Bryson AL, Wengenack NL, Sampathkumar P (2019) Fungal diagnostic stewardship in bronchoscopy specimens for immunocompetent patients in the intensive care unit. Mayo Clin Proc 94(9):1781-1785. https://doi.org/10. 1016/j.mayocp.2019.02.020

17. Martinelli AW, Patil P, Wong VK, Enoch DA, Sander CR (2019) A positive BAL galactomannan in non-haemato-oncology patients risks harmful overtreatment. J Med Microbiol 68(12):1766-1770. https://doi.org/10.1099/jmm.0.001109

Publisher's Note Springer Nature remains neutral with regard to jurisdictional claims in published maps and institutional affiliations. 